\title{
Inhibition of a novel fibrogenic factor Tl1a reverses established colonic fibrosis
}

\author{
DQ Shih ${ }^{1,4}$, L Zheng ${ }^{1,2,4}$, X Zhang ${ }^{2}$, H Zhang ${ }^{1,2}$, Y Kanazawa $^{1}$, R Ichikawa $^{1}$, KL Wallace ${ }^{1}, \mathrm{~J} \mathrm{Chen}^{1}$, \\ C Pothoulakis ${ }^{3}$, HW Koon ${ }^{3}$ and SR Targan ${ }^{1}$
}

Intestinal fibrostenosis is among the hallmarks of severe Crohn's disease. Patients with certain TNFSF15 (gene name for TL1A) variants over-express TL1A and have a higher risk of developing strictures in the small intestine. In addition, sustained Tl1a expression in mice leads to small and large intestinal fibrostenosis under colitogenic conditions. The aim of this study was to determine whether established murine colonic fibrosis could be reversed with Tl1a antibody (Ab). Treatment with neutralizing TI1a Ab reversed colonic fibrosis back to the original pre-inflamed levels, potentially as a result of lowered expression of connective tissue growth factor, II31Ra, transforming growth factor $\beta 1$ and insulin-like growth factor-1. In addition, blocking Tl1 a function by either neutralizing Tl1a Ab or deletion of death domain receptor 3 (Dr3) reduced the number of fibroblasts and myofibroblasts, the primary cell types that mediate tissue fibrosis. Primary intestinal myofibroblasts expressed Dr3 and functionally responded to direct Tl1a signaling by increasing collagen and II31Ra expression. These data demonstrated a direct role for TL1A-DR3 signaling in tissue fibrosis and that modulation of TL1A-DR3 signaling could inhibit gut fibrosis.

\section{INTRODUCTION}

Crohn's disease (CD) is a chronic inflammatory condition with pathological features such as patchy transmural inflammation and fibrostenosis. It is accepted that chronic intestinal inflammation leads to the development of fibrosis. However, even when treated with potent anti-inflammatory therapies, $\sim 20 \%$ of $\mathrm{CD}$ patients still develop stricturing complications that require surgical intervention. ${ }^{1,2}$ A study using the Salmonella typhimurium colitis model showed that despite the attenuation of intestinal inflammation with antibiotic treatment, fibrosis not only persisted, but actually progressed and that myofibroblast activation and fibrogenesis were not completely resolved by early removal of the inflammatory trigger. ${ }^{3}$ Several other studies have shown that pathways independent of inflammation also drive fibrosis, ${ }^{4-6}$ and that removal of the inciting inflammatory stimulus does not reverse established fibrosis.

TL1A (a protein encoded by TNFSF15) is a member of the tumor necrosis factor (TNF) superfamily that binds to death domain receptor 3 (DR3) and modulates the adaptive immune response. ${ }^{7}$ A particular TNFSF15 haplotype is associated with higher TL1A expression, increased risk of $\mathrm{CD}$, intestinal fibrostenosis, and greater need for surgery. ${ }^{8-11}$ In addition to human reports, studies in mice also implicate the Tl1a/Dr3 signaling pathway in mucosal inflammation and fibrosis. Previously, constitutive TI1 a expression in mice were shown to have spontaneous ileitis and increased collagen deposition. ${ }^{12-15}$ Under colitogenic conditions, Tl1a transgenic mice develop worsened small and large intestinal inflammation and fibrostenosis. $^{10}$

Tlla antibody (Ab) has been shown to prevent and treat murine dextran sodium sulfate (DSS) colitis; ${ }^{16}$ however, whether targeting Tlla independently reduces gut fibrosis has not been established. In the present study, we used two distinct chronic colitis models, DSS and adoptive T-cell transfer, to determine whether the reversal of colonic fibrosis subsequent to treatment with Tlla $\mathrm{Ab}$ was independent of its previously reported effect in amelioration of inflammation.

\footnotetext{
${ }^{1}$ F. Widjaja Foundation, Inflammatory Bowel and Immunobiology Research Institute, Cedars-Sinai Medical Center, Los Angeles, California, USA. ${ }^{2}$ Department of Gastroenterology, The Second Hospital of Hebei Medical University, Hebei, China and ${ }^{3}$ IBD Center, David Geffen School of Medicine, UCLA, Los Angeles, California, USA. Correspondence: SR Targan (Stephan.Targan@cshs.org)

${ }^{4}$ The first two authors shared co-first authorship.
} 
We found that the anti-fibrotic effect of was associated with reversal of the fibrogenic program, leading to reduced numbers of fibroblasts and myofibroblasts. Further, to determine whether the fibrogenic effect of Tlla was through direct signaling of intestinal fibroblasts, we generated mice that were deficient of Dr3 $\left(D r 3^{-/-}\right)$, the only known receptor for Tl1a. Dr $3^{-/-}$ mice had significantly fewer intestinal fibroblasts, and showed that Dr3 was preferentially expressed on intestinal myofibroblasts. Furthermore, Tl1a directly increased collagen and Il31Ra expression on wild type (WT) but not $D r 3^{-/-}$intestinal fibroblasts. These findings indicate that the TL1A/DR3 signaling pathway blockade may constitute a novel therapeutic approach for the treatment of $\mathrm{CD}$, not only by inhibiting inflammation, but also directly reducing fibrogenesis and reversal of established fibrosis.

\section{RESULTS}

\section{Tl1a Ab reversed established colonic fibrosis}

Mice with constitutive Tlla expression were previously shown to develop increased gut fibrosis. ${ }^{10,14,15}$ Therefore, to determine the effect of blocking Tl1a signaling on colonic fibrosis, we used two mouse models of chronic colitis; adoptive T-cell transfer and chronic DSS. In the adoptive T-cell transfer model, immune-deficient $R a g 1^{-1-}$ mice were adoptively transferred with naïve $\mathrm{CD} 4{ }^{+} \mathrm{CD} 45 \mathrm{RB}^{\text {hi }} \mathrm{T}$ cells. Tl1a $\mathrm{Ab}\left(80 \mathrm{mg} \mathrm{kg}^{-1}\right)$ or isotype control $\mathrm{Ab}$ (Iso $\mathrm{Ab}, 80 \mathrm{mg} \mathrm{kg}^{-1}$ ) was administered two times per week beginning on day 29 (week 4) post-transfer when colitis was established (Figure 1a, left panel). Sirius red stain was used to measure the degree of collagen deposition. By the 4th week after naive T-cell transfer, increased collagen deposition was found in the colons of mice in the Pre-Tx group compared with the baseline Rag Co group (Figure 1b, left and middle panels). The degree of collagen deposition in the colon was greater by the 8th week in mice receiving control Iso $\mathrm{Ab}$. Treatment with Tlla Ab led to a significant reduction in collagen deposition compared with mice that received the Iso $\mathrm{Ab}$ or the Pre-Tx groups (Figure 1b, left and middle panels). Notably, collagen deposition was not significantly different when the Tl1a-treated mice were compared with normal Rag Co mice (Figure 1b, left and middle panels). The Sircol assay, a dye-binding method designed to quantitatively measure acid and pepsin-soluble collagen, was used to measure colonic collagen and which showed increased soluble collagen in the Pre-Tx group compared with the Rag Co group (Figure 1b, right panel). Addition of control Iso $\mathrm{Ab}$ led to further increase in soluble collagen, whereas Tlla $\mathrm{Ab}$ administration reduced soluble collagen to levels similar to the baseline Rag Co group (Figure 1b, right panel).

In the chronic DSS model, Tl1a (20 $\left.\mathrm{mg} \mathrm{kg}^{-1}\right)$ or isotype $\mathrm{Ab}$ (20 $\mathrm{mg} \mathrm{kg}^{-1}$ ) was administered twice a week beginning at day 15 when colitis was established (Figure 1a, right panel). Reduction in collagen deposition and soluble collagen in the colon with Tlla Ab treatment was observed when compared with the Iso $\mathrm{Ab}$ and the Pre-Tx groups (Figure 1c). Together, these data indicated that blocking Tl1a signaling not only prevented further accumulation of collagen but also reversed collagen to similar levels measured before the onset of inflammation.

\section{Tl1a Ab administration reduced but did not completely reverse established colitis}

We next sought to determine whether the reversal of collagen levels seen with blocking Tl1a signaling by Tl1a Ab would be paralleled by a like reduction of clinical and histologic colonic inflammation. In both chronic colitis models, administration of Tlla Ab led to a significant lowering of disease activity index (DAI) as compared with the Iso Ab group (Figure 2a). However, while Tl1a Ab reduced collagen deposition back to the baseline, the decrease in disease activity, as measured by DAI, did not reach baseline levels and that clinical parameters of inflammation persisted (weight loss, loose stool, and stool blood).

Histological examination of the colon revealed reduced inflammation characterized by reduced cellular infiltrate, mucin depletion, crypt abscesses, muscularis propria thickness, and architectural changes with Tl1a Ab therapy compared with Iso $\mathrm{Ab}$ group in both the adoptive transfer and chronic DSS colitis models (Figure $\mathbf{2 b}, \mathbf{c}$ ). The reduction in colonic histological inflammation was also significant compared with the Pre-Tx group in both models of chronic colitis (Figure $\mathbf{2 b , c}$ ). Although histologic inflammation was reduced with Tlla $\mathrm{Ab}$ treatment, colonic inflammation was still significantly higher compared with baseline in the WT control (Co) group in the chronic DSS colitis model (Figure 2c).

Given that there was still significantly elevated clinical disease activity in both models and that in the DSS model there was worsened histologic colitis as compared with the WT Co despite treatment with Tl1a Ab, the reversal of collagen deposition is consistent with at least in part, a direct fibrosisameliorating effect of Tl1a Ab.

\section{Blocking TI1a-Dr3 signaling reduced numbers of intestinal fibroblasts and myofibroblasts}

Colonic myofibroblasts are a cell population involved in gut fibrogenesis. ${ }^{17}$ To study the cellular mechanisms of collagen deposition reduction with Tlla Ab, fibroblast expression of vimentin and myofibroblast coexpression of vimentin and alpha smooth muscle actin ( $\alpha \mathrm{SMA}$ ) were measured to assess the numbers of these cell types. After naïve T-cell transfer in both the Pre-Tx and Iso Ab groups, the numbers of colonic fibroblasts and myofibroblasts were increased (Figure 3a). However, treatment with Tlla Ab led to a reduction in the number of fibroblasts and myofibroblasts to levels similar to normal Rag Co (Figure 3a).

In the chronic DSS model, mice treated with Tl1a Ab exhibited a similar reduction in the number of colonic fibroblasts and myofibroblasts compared with the Iso or the Pre-Tx groups (Figure 3b). Consistent with what was observed in the adoptive transfer model, the number of gut fibroblasts and myofibroblasts with Tlla Ab treatment reduced to a level that was not statistically different from WT baseline control (Figure 3b). Because there was still significantly worsened colitis with Tlla $\mathrm{Ab}$ treatment as compared with WT Co group in the chronic 
a

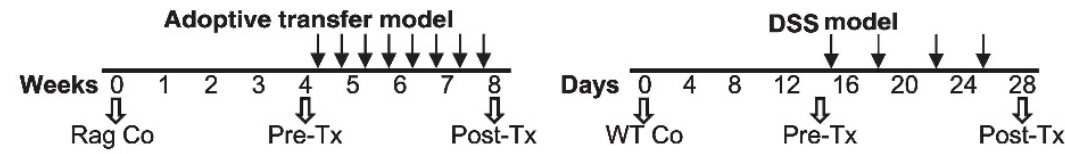

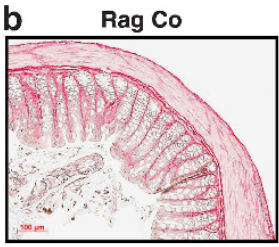

Isotype Ab

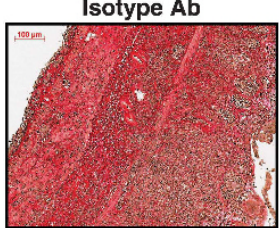

WT Co

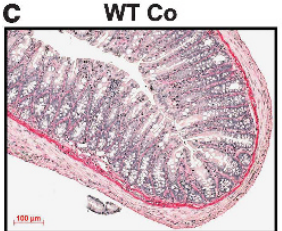

Isotype Ab

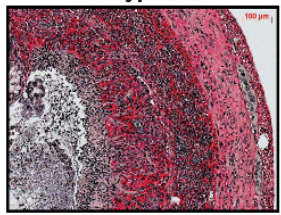

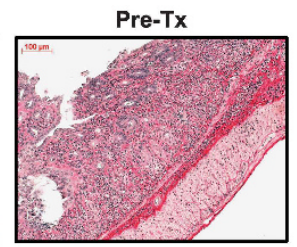

TI1a Ab

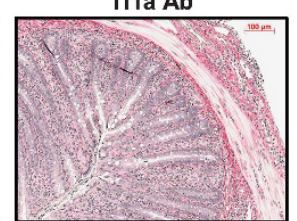

Pre-Tx

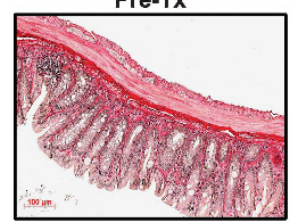

TI1a Ab

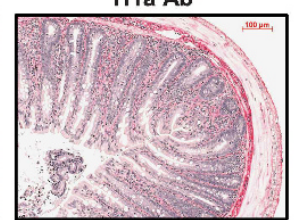

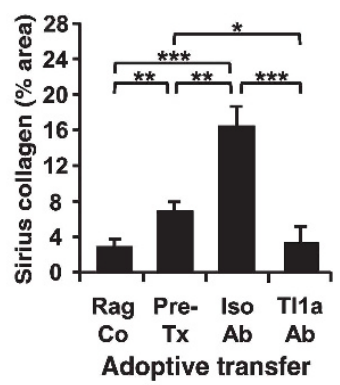
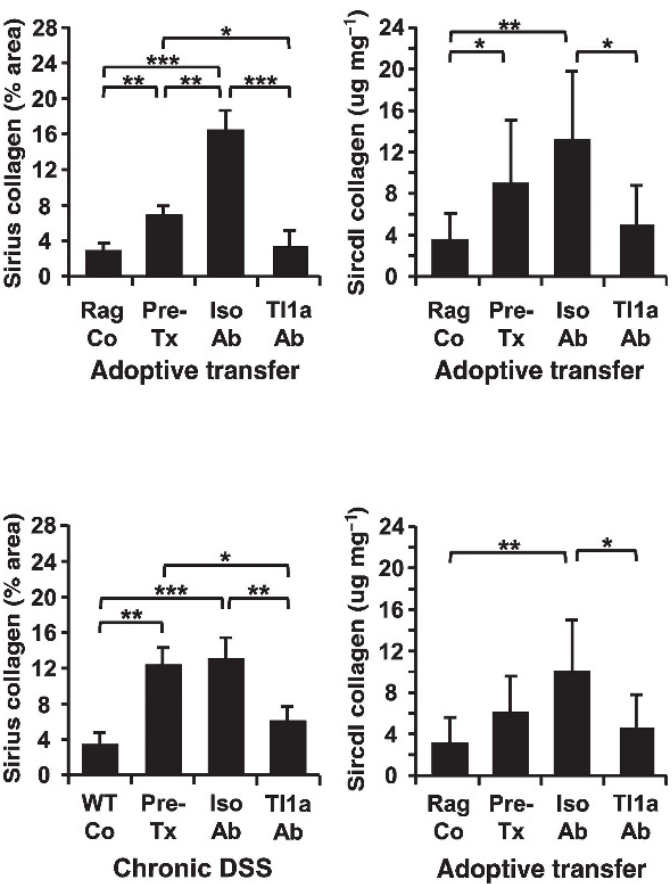

Figure 1 Reversal of established fibrosis with TI1a antibody (Ab) therapy. (a) TI1a Ab treatment schematics for the adoptive transfer model (left panel) and the chronic dextran sodium sulfate (DSS) colitis model (right panel); baseline control mice (Rag $n=5$ or wild type (WT) Co $n=5$ ), pre-treatment group (Pre-Tx, $n=5$ for transfer, $n=6$ for DSS), posttreatment group (Post-Tx, $n=7-14$ ). Representative Sirius red staining of collagen deposition in mid-colon tissue sections at $\times 100$ magnification is shown for adoptive transfer model in $\mathbf{b}$ (left panels) and chronic DSS model in $\mathbf{c}$ (left panels). Percent of colon with collagen staining was quantitated and expressed as mean \pm s.d. for the adoptive transfer model in $\mathbf{b}$ (middle panel) and for the chronic DSS model in $\mathbf{c}$ (middle panel). Quantitation of soluble collagen from the colon were determined and expressed as mean \pm s.d. for the adoptive transfer model in $\mathbf{b}$ (right panel) and for the chronic DSS model in c (right panel). At least 20 independent fields per group were scored and data are expressed as mean \pm s.d. ${ }^{\star} P<0.05,{ }^{* *} P<0.01,{ }^{* \star *} P<0.001$.

DSS colitis model, the reduced numbers of myofibroblasts and fibroblasts is consistent with at least in part, a direct consequence of neutralizing Tl1a, rather than solely a secondary effect through reduced inflammation.

We next assessed whether there were Dr3 expression changes in association with the fibrotic changes in these murine models of chronic colitis. Immunofluorescent staining revealed increased Dr3 expression in the Pre-Tx and Iso Ab groups as compared with both baseline control groups (Rag Co and WT Co) and the Tlla Ab-treated groups in both the adoptive transfer and chronic DSS colitis models (Figure 3c,d). Notably, there was an expression of Dr3 in a percentage of fibroblasts in the Pre-Tx and Isotype Ab groups (Figure $3 \mathbf{c}$ and $\mathbf{d}$ ). Real-time quantitative reverse transcriptase-PCR analysis showed that the expression of Dr3 was significantly higher in the Iso Ab group as compared with mice in the both baseline control (Rag Co and WT Co) and Tlla Ab treatment groups in both models (Figure 3e). In addition, Tlla mRNA expression was significantly increased in the Iso $\mathrm{Ab}$ group as compared with un-inflamed controls (Rag Co and WT Co) and the Tlla Ab treatment groups in both the adoptive transfer and chronic DSS colitis models (Figure 3f). These results are consistent with a direct relationship between Dr3-Tl1a expression and increase in intestinal fibrosis.

To determine whether the reduction in the number of intestinal fibroblasts and myofibroblasts could be due to direct Tl1a-Dr3 signaling, we generated Dr3-deficient $\left(\operatorname{Dr} 3^{-/-}\right)$ mice (Supplementary Figure S1A, B online). Although there was no spontaneous colitis in either WT or Dr3 $3^{-/-}$mice up to 8 weeks of age (Figure 4a, top panel), there were significantly fewer intestinal fibroblasts in $D r 3^{-/-}$as compared with WT littermate mice as shown by immunofluorescent staining of vimentin (Figure 4a, middle panel) and quantitation of the total recovered fibroblasts per colon (Figure 4a, bottom panel). There were no morphological differences between WT and Dr3 ${ }^{-/-}$fibroblasts by immunofluorescent staining with 

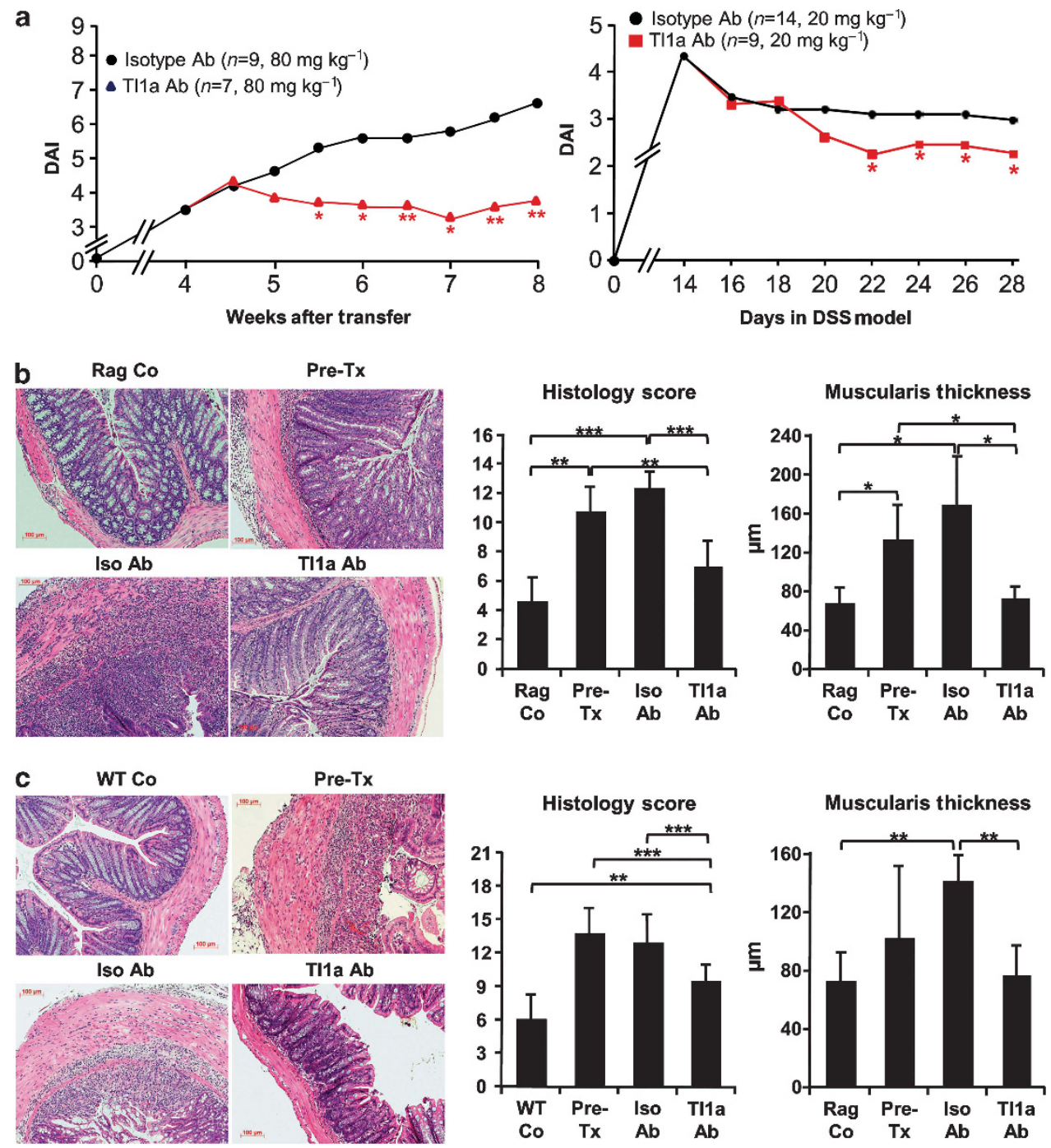

Figure 2 Tl1a antibody $(\mathrm{Ab})$ reduced established chronic colitis. (a) Disease activity index (DAI) of the adoptive transfer (left panel) and chronic dextran sodium sulfate (DSS) colitis model (right panel) is compared between isotype Ab- and Tl1a Ab-treated groups. Representative hematoxylin and eosinstained mid-colon sections from the adoptive transfer model (b) and chronic DSS colitis model (c) at $\times 100$ magnification are shown and quantitative histology scores ( $\mathbf{b}$ and $\mathbf{c}$, middle panels) and thickness of muscularis propria (b and $\mathbf{c}$, right panels) are shown. At least 20 independent fields per group are scored and data are expressed as mean \pm s.d. ${ }^{\star} P<0.05,{ }^{* \star} P<0.01,{ }^{* \star \star} P<0.001$.

vimentin and $\alpha$ SMA (Figure 4a, middle panel) or with light microscopy (Figure 4a, bottom panel). Ex vivo CellTrace Violet assay and Annexin $\mathrm{V}$ stain were used to determine whether the difference in the numbers of intestinal fibroblasts between WT and $\mathrm{Dr}^{-/-}$mice was due to proliferation and/or apoptosis, respectively. Flow cytometric analysis showed similar rates of proliferation as evidenced by the overlapping CellTrace Violet intensity between WT and $D r 3^{-1-}$ intestinal fibroblasts (Figure 4b). No differences were observed in the rate of apoptosis between WT and $D r 3^{-1-}$ intestinal fibroblasts (Figure 4c).

\section{Reversal of fibrogenesis with TI1a Ab administration}

To study the molecular mechanisms of reversal of established intestinal fibrosis with Tlla $\mathrm{Ab}$ treatment, we measured the expression of collagen, Il31 receptor (Il31Ra), fibrogenic program mediators (transforming growth factor $\beta 1$ (Tgf $\beta 1$ ), connective tissue growth factor (Ctgf), insulin-like growth factor-1 (Igf1), and Pten), and factors (metalloprotease (Mmp) and tissue inhibitor of metalloprotease (Timp)) involved in extracellular matrix (ECM) remodeling. ${ }^{17,18}$ Lower levels of collagen expression were found in both the adoptive transfer (Table 1) and chronic DSS (Table 2) models. Tl1a Ab treatment resulted in normalization of the fibrogenic program mediators as evidenced by lower expression of Tgf $\beta 1$ and Igf1 in the adoptive transfer (Table 1) and Tgf $\beta 1$ in the chronic DSS (Table 2) models. Il31Ra, a gene known to be expressed on fibroblasts, also appeared to be regulated by Tl1a (Tables 1 and 2). The expression of Ctgf, a downstream mediator of $\operatorname{Tgf} \beta$ signaling, was reduced with Tlla $\mathrm{Ab}$ administration as compared with Pre-Tx and Iso Ab groups in the adoptive transfer model (Table 1). 
a

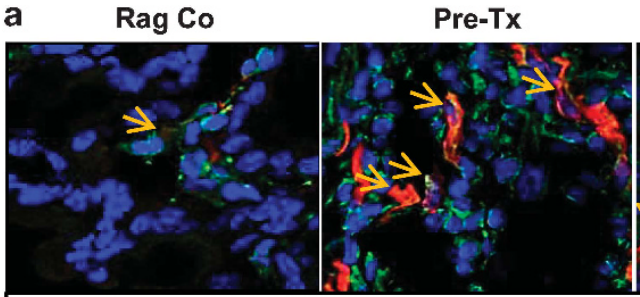

Isotype Ab

TI1a Ab

Blue - DAPI

Green - vimentin
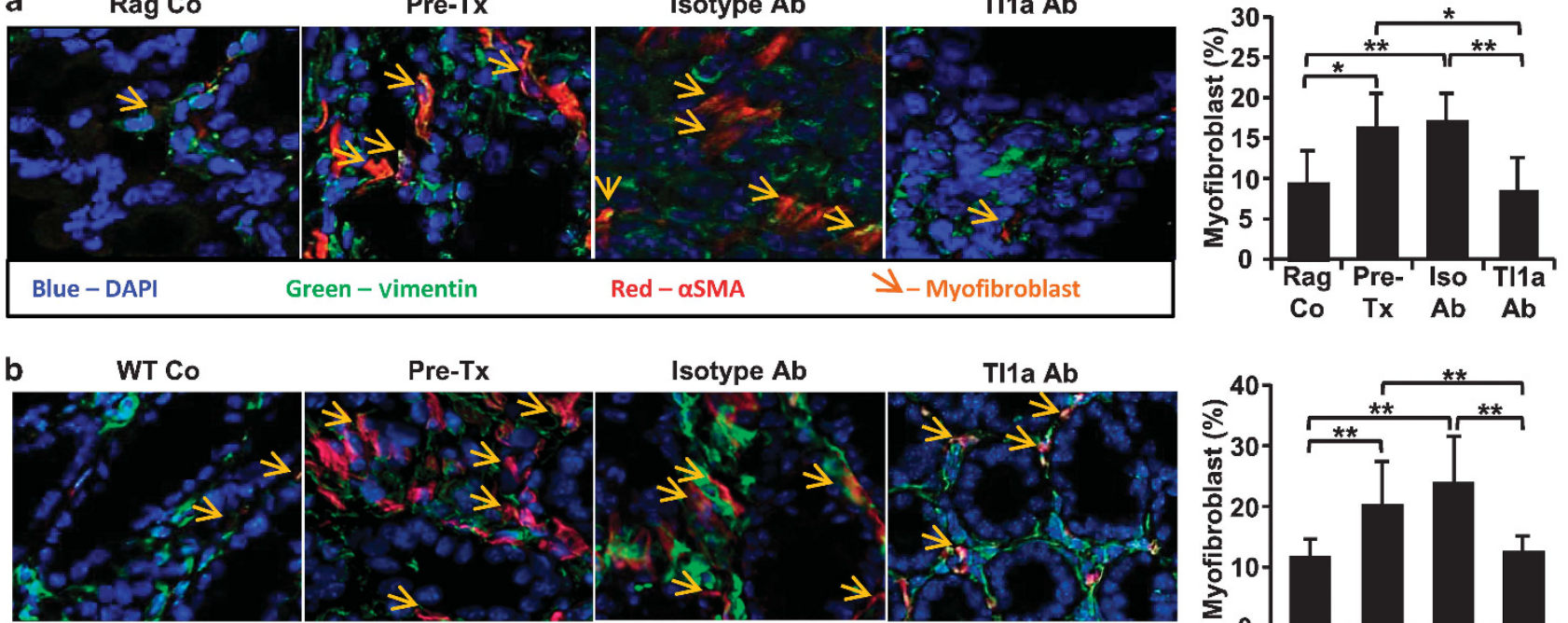

Red-aSMA

$\nu_{-}$Myofibroblast

Blue - DAPI

Green - vimentin

Isotype Ab

TI1a Ab
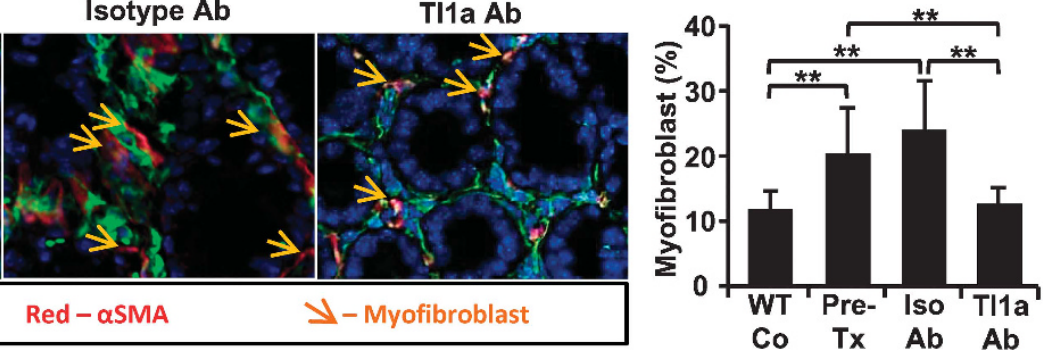

C Rag Co

Pre-Tx

Red - aSMA

U-Myofibroblast

Co $\mathrm{Tx}$ Ab $\mathrm{Ab}$
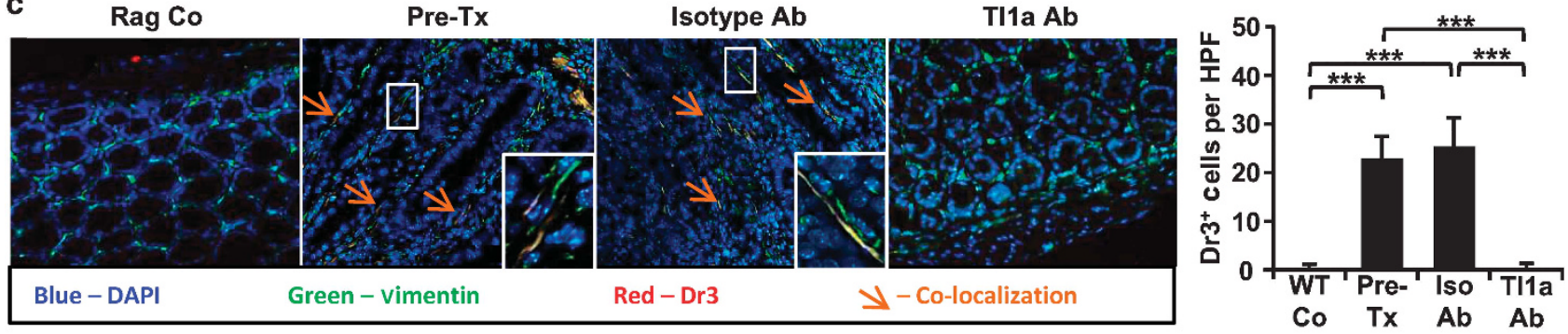

d WT Co

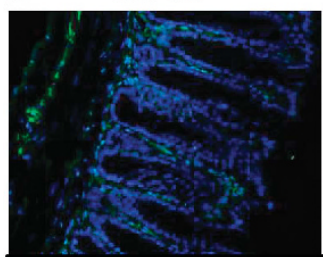

Pre-Tx

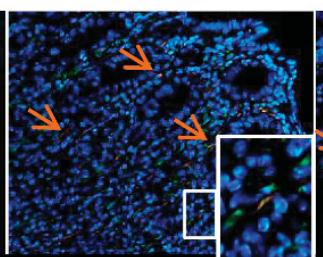

Green - vimentin
Isotype $\mathbf{A b}$

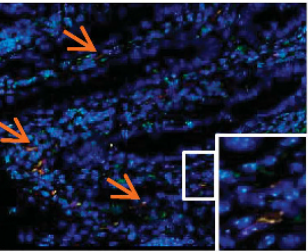

Red-Dr3

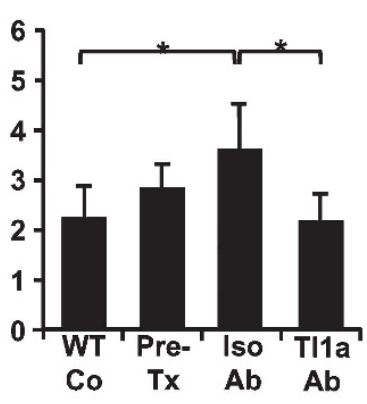

Chronic DSS

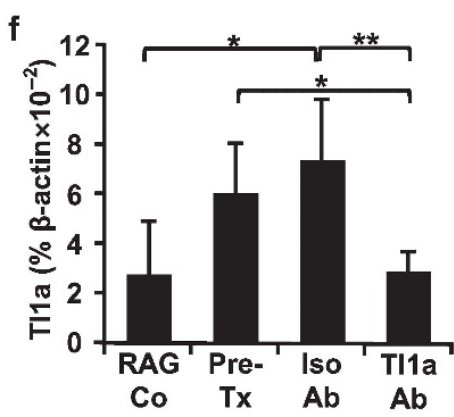

Adoptive transfer
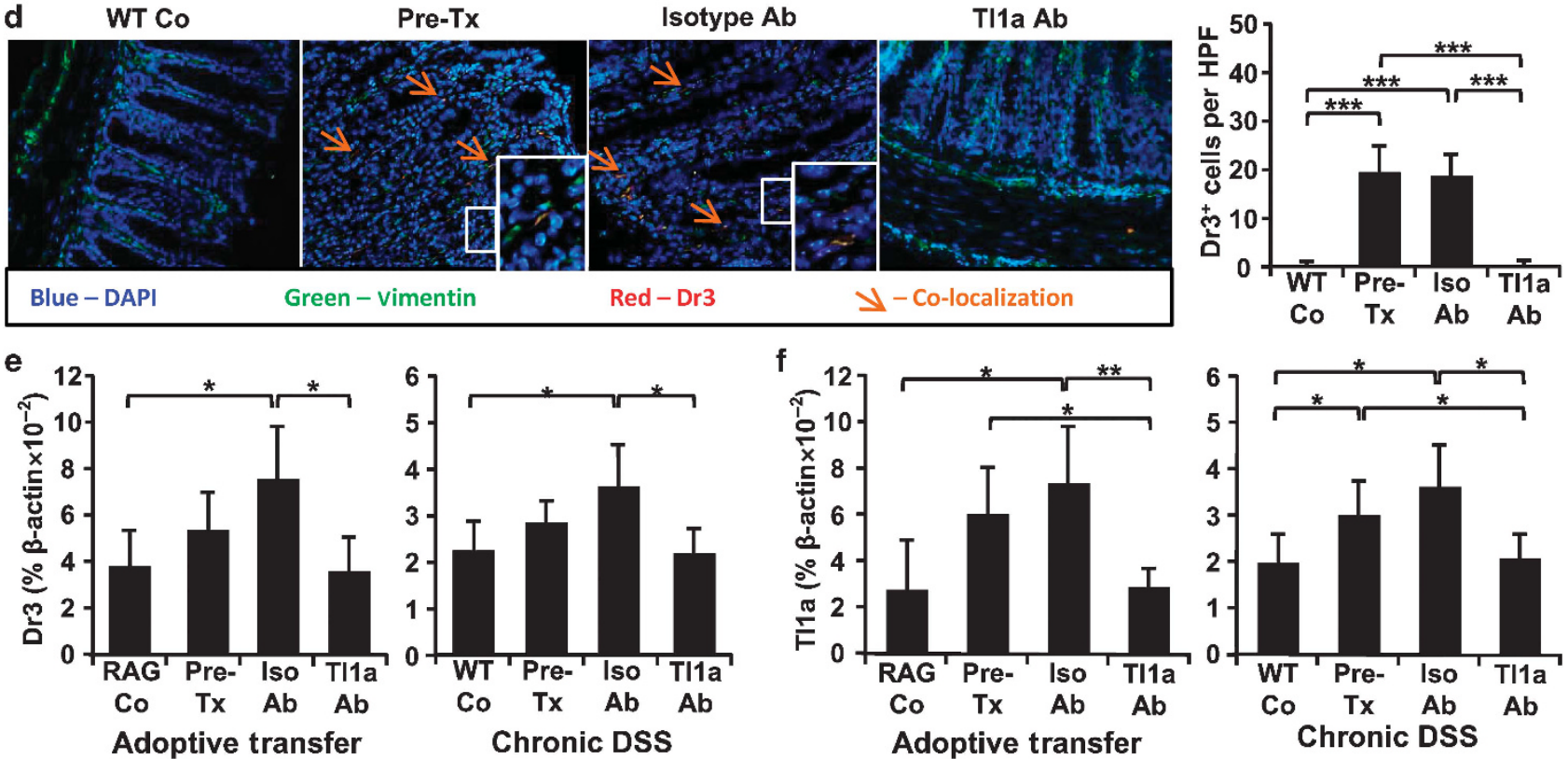

Figure 3 Tl1a antibody $(\mathrm{Ab})$ reduced myofibroblast number and expression of death domain receptor 3 (Dr3) and TI1a. Representative immunofluorescent staining of vimentin (green) and alpha smooth muscle actin ( $\alpha \mathrm{SMA}$ ) (red) from mid-colon sections from the adoptive transfer model (a) and chronic dextran sodium sulfate (DSS) model (b) at $\times 630$ magnification is shown. Orange arrows denote myofibroblasts that co-express vimentin and $\alpha$ SMA. Percentages of myofibroblasts from the mid-colon sections were quantitated and expressed as mean $\pm \mathrm{s}$.d. for the adoptive transfer model (a, right panel) and chronic DSS model (b, right panel). At least 10 independent fields were scored per group for $\mathbf{a}$ and $\mathbf{b}$. Representative immunofluorescent staining of vimentin (green) and Dr3 (red) from mid-colon sections is shown from the adoptive transfer model (c) and chronic DSS model (d). Figure insets for (c) and (d) are larger view of the images that were acquired at $\times 200$ magnification. At least eight independent fields were quantitated per group and plotted as Dr $3^{+}$cells per high power field (HPF). Colonic Dr3 (e) and TI1a (f) mRNA was quantitated and shown as mean \pm s.d. $(n=5-14)$. Tl1 a Ab-treated groups are compared with baseline Rag Co, wild type (Wt) Co, Pre-Tx, and Iso Ab experimental groups. ${ }^{\star} P<0.05$, ${ }^{\star \star} P<0.01$, ${ }_{\star * *} P<0.001$. 
a
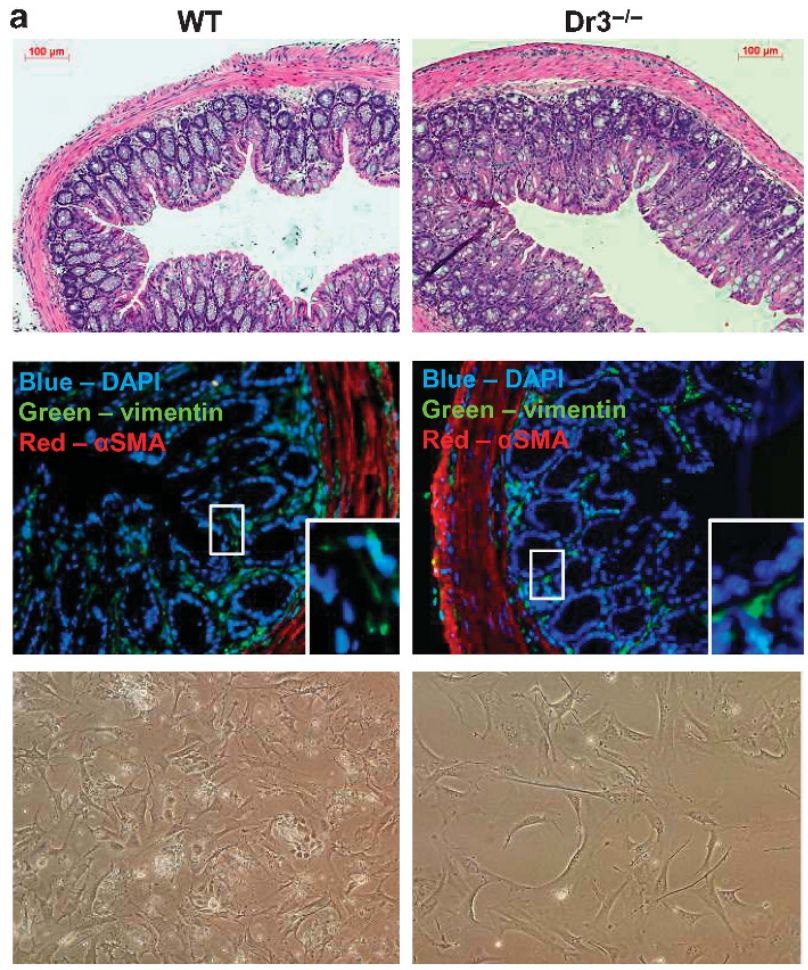

Dr3-l-
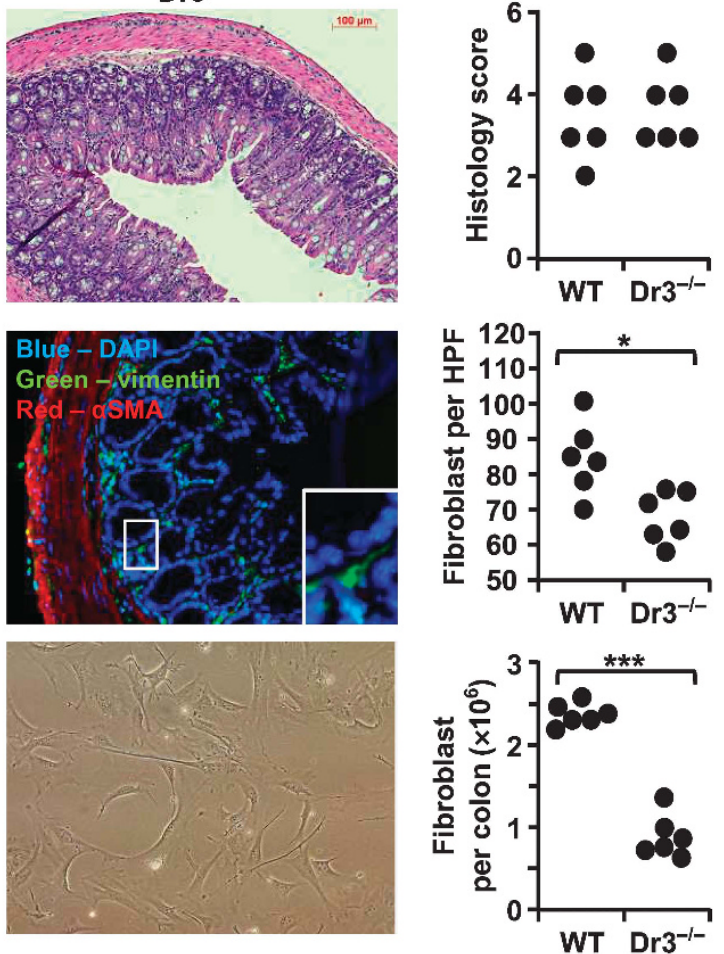
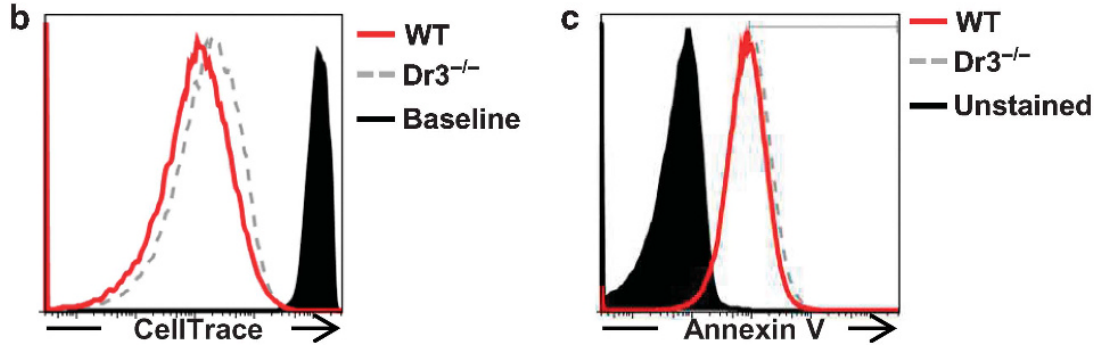

Figure 4 Reduced intestinal fibroblasts with death domain receptor 3 (Dr3) deficiency. (a) Representative hematoxylin and eosin-stained colon at $\times 100$ magnification with quantitation of inflammation is shown on the upper panels. Representative Vimentin/alpha smooth muscle actin ( $\alpha$ SMA)-stained colon at $\times 200$ magnification (insets are larger view at $\times 200$ magnification) with quantitation of fibroblasts per high power field (HPF) is shown in the middle panels. Representative photographs of intestinal fibroblasts recovered from littermate wild type (WT) and Dr3 ${ }^{-/-}$colon and individual total fibroblasts per colon are shown (a, bottom panels). Representative flow cytometric histograms of proliferating fibroblasts (b) and fibroblasts undergoing apoptosis (c) from WT and Dr3 $3^{-1-}$ mice are shown. Decreased CellTrace violet fluorescence intensity indicates proliferation. Increased Annexin V staining indicates apoptosis. Representative flow cytometric histograms of at least six independent experiments with similar results are shown. ${ }^{\star} P<0.05$, ${ }^{* \star *} P<0.001$

ECM remodeling was assessed by measuring the expression of Mmp and Timp. Compared with the isotype Ab group, the expression of genes involved in ECM degradation was reduced in mice treated with Tlla Ab in the adoptive transfer model (Mmp2, Mmp3; Table 1) and in the chronic DSS model (Mmp2, Mmp3, Mmp13; Table 2). Notably, the expression of Timp was lower with Tlla Ab treatment in the adoptive transfer model (Timp2, Table 1) and in the chronic DSS model (Timp1, Timp2; Table 2). Although Mmp expression was decreased, it is possible that reduction in Timp expression resulted in a net increase in Mmp activity that led to enhanced removal of established ECM components rather than induction of tissue damage. These results demonstrate that treatment with Tlla Ab reduced the fibrogenic program, leading to decreased collagen synthesis.

\section{Intestinal fibroblasts express Dr3 and respond to TI1a stimulation}

To determine whether intestinal fibroblasts functionally respond to direct Tl1a signaling, mRNA levels of Dr3 were measured and found to be expressed at low levels in WT $(0.0018 \pm 0.001 \% \beta$-actin $)$ but undetectable in Dr3-deficient primary intestinal fibroblasts. We next performed flow cytometric analysis to determine whether Dr3 was expressed on vimentin ${ }^{+} \alpha_{\mathrm{SMA}}{ }^{-}$fibroblasts or vimentin ${ }^{+} \alpha \mathrm{SMA}^{+}$ myoflbroblasts. Our results showed that Dr3 was expressed 
Table 1 Expression analysis of fibrosis mediators in the adoptive transfer colitis model

\begin{tabular}{|c|c|c|c|c|c|c|c|}
\hline & Baseline & & Iso Ab & & $11 a \mathrm{Ab}-8$ & $g^{-1}$ & \\
\hline & $n=6$ & $n=6$ & $n=7$ & $n=6$ & Rag & Pre-Tx & Iso $A b$ \\
\hline col1a1 & $0.19 \pm 0.12$ & $0.19 \pm 0.12$ & $0.21 \pm 0.10$ & $0.11 \pm 0.03$ & NS & 0.024 & 0.03 \\
\hline col1a2 & $0.49 \pm 0.29$ & $0.76 \pm 0.32$ & $1.23 \pm 0.78$ & $0.39 \pm 0.12$ & NS & 0.024 & 0.026 \\
\hline col3a1 & $12.69 \pm 3.61$ & $16.45 \pm 3.93$ & $16.08 \pm 4.04$ & $9.66 \pm 3.44$ & NS & 0.0073 & 0.014 \\
\hline $\operatorname{Tgf} \beta 1$ & $0.16 \pm 0.06$ & $0.40 \pm 0.16$ & $0.50 \pm 0.17$ & $0.25 \pm 0.06$ & 0.018 & 0.046 & 0.003 \\
\hline Ctgf & $0.66 \pm 0.13$ & $1.04 \pm 0.40$ & $1.04 \pm 0.32$ & $0.54 \pm 0.08$ & NS & 0.021 & 0.007 \\
\hline lgf1 & $0.32 \pm 0.06$ & $0.53 \pm 0.18$ & $0.73 \pm 0.36$ & $0.41 \pm 0.15$ & NS & NS & 0.047 \\
\hline Pten & $3.80 \pm 0.75$ & $2.28 \pm 0.53$ & $1.86 \pm 0.25$ & $2.03 \pm 0.67$ & 0.0015 & NS & NS \\
\hline II31Ra & $0.003 \pm 0.001$ & $0.005 \pm 0.002$ & $0.007 \pm 0.003$ & $0.004 \pm 0.001$ & NS & NS & 0.034 \\
\hline Timp1 & $0.038 \pm 0.014$ & $0.19 \pm 0.24$ & $0.18 \pm 0.12$ & $0.14 \pm 0.07$ & 0.016 & NS & NS \\
\hline Timp2 & $1.11 \pm 0.27$ & $0.86 \pm 0.15$ & $0.79 \pm 0.12$ & $0.59 \pm 0.18$ & 0.001 & 0.001 & 0.048 \\
\hline
\end{tabular}

$\mathrm{Ab}$, antibody; ctgf, connective tissue growth factor; Igf1, insulin-like growth factor-1; $\mathrm{mmp}$, metalloprotease; NS, not significant; timp, tissue inhibitors of metalloprotease; tgf $\beta 1$, transforming growth factor $\beta 1$.

Table 2 Expression analysis of fibrosis mediators in the dextran sodium sulfate model

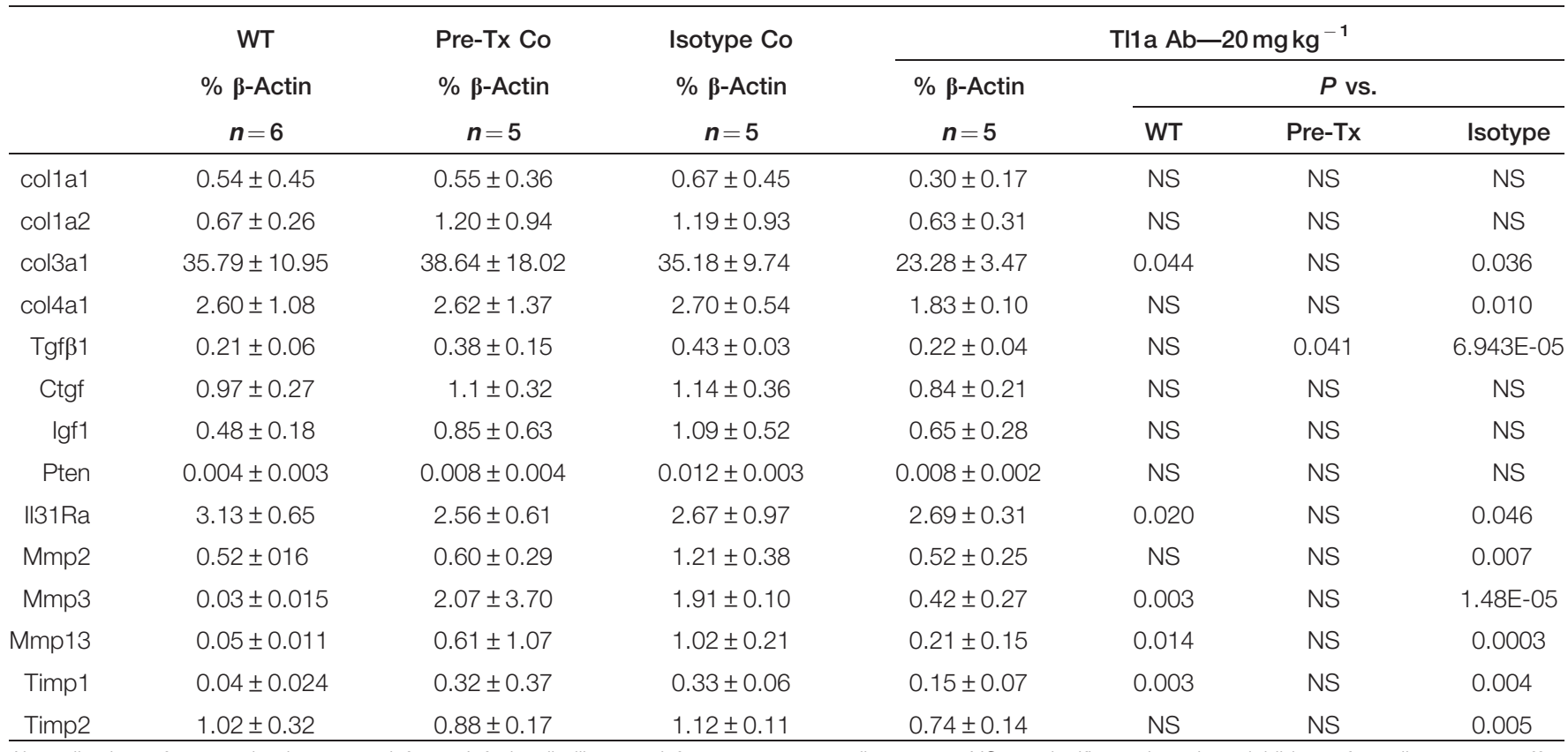

$\mathrm{Ab}$, antibody; ctgf, connective tissue growth factor; Igf1, insulin-like growth factor-1; mmp, metalloprotease; NS, not significant; timp, tissue inhibitors of metalloprotease; tgf $\beta 1$, transforming growth factor $\beta 1$; WT, wild type.

preferentially on vimentin ${ }^{+} \alpha \mathrm{SMA}^{+}$myoflbroblasts as compared with vimentin ${ }^{+} \alpha \mathrm{SMA}^{-}$fibroblasts (Supplementary Figure S1C). In addition, there was a direct correlation of Dr3 expression with $\alpha$ SMA levels on myofibroblasts; with a higher proportion of Dr3 expression on myofibroblasts with the highest aSMA expression (Figure 5a). In addition, sorted aSMA-positive primary intestinal fibroblasts that were immunostained with $\alpha \mathrm{SMA}$ and Dr3 showed co-staining of 


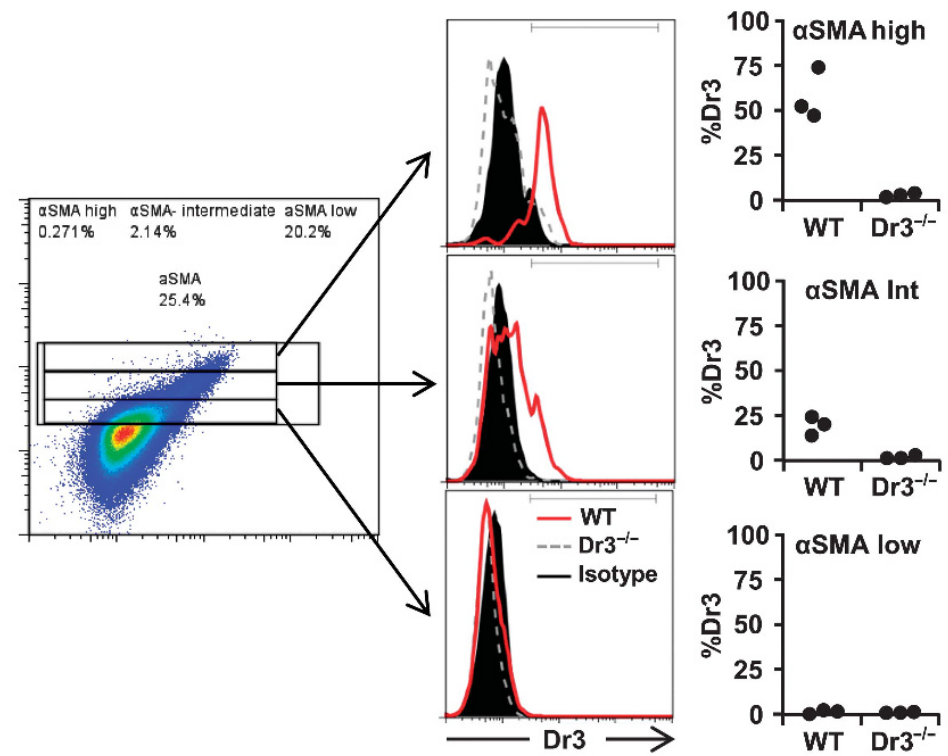

b

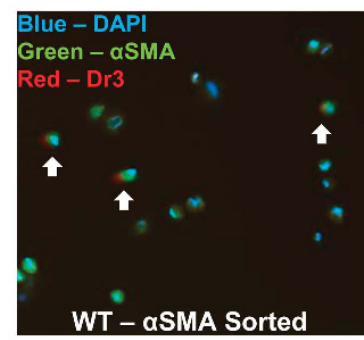

c

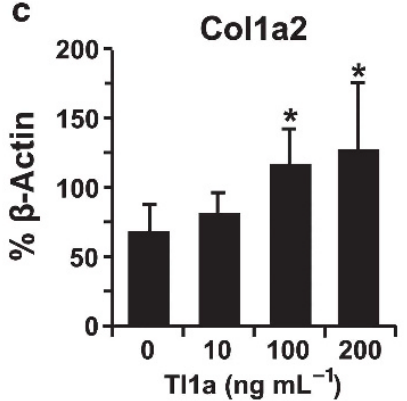

d

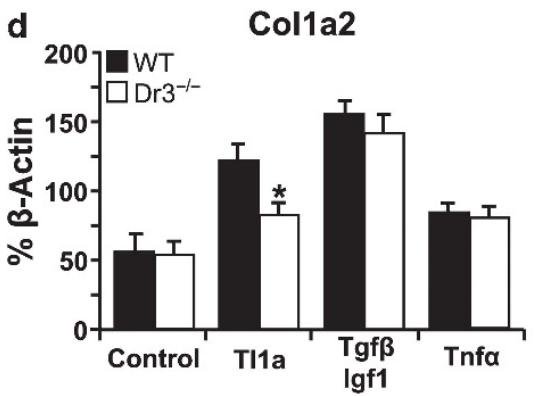

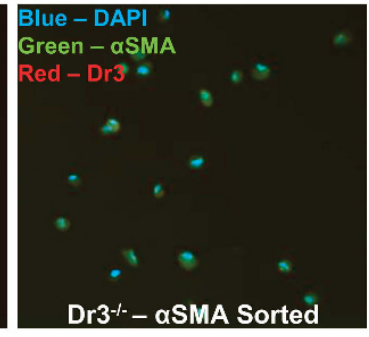

II31Ra

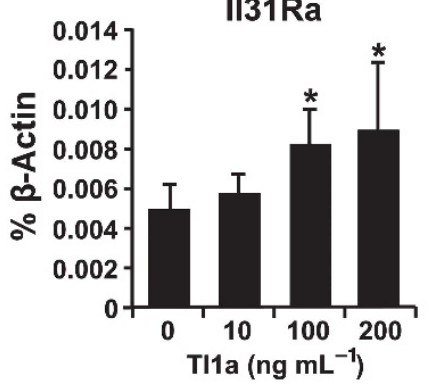

II31Ra

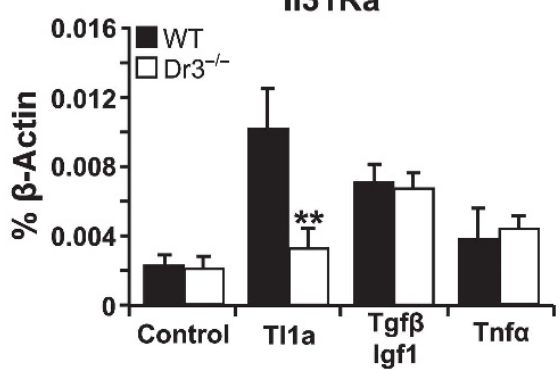

Figure 5 Intestinal fibroblasts express death domain receptor 3 (Dr3) and respond to Tl1a stimulation. (a) Primary intestinal fibroblasts were stained with Dr3, alpha smooth muscle actin ( $\alpha$ SMA), and vimentin and analyzed by flow cytometry. Fibroblasts expressing high, intermediate, and low $\alpha$ SMA were gated as shown and Dr3 staining is preferentially found in $\alpha$ SMA high $>$ intermediate $>$ low. Three independent experiments were performed. (b) Data are representative of three independently sorted $\alpha$ SMA-positive myofibroblasts at $\times 200$ magnification. There was co-staining of Dr3 in wild type (WT), but not in Dr3-deficient $\alpha$ SMA-positive myofibroblasts. (c) Expression of Col1a2 and II31Ra mRNA in WT primary intestinal fibroblasts with increasing TI1a stimulation (0-200 $\mathrm{ng} \mathrm{ml}^{-1}$ ) and represented as mean \pm s.d. are shown $(n=3)$. (d) Induction of Col1a2 and II31Ra mRNA by TI1a, transforming growth factor $\beta 1$ (Tgf $\beta$ )/insulin-like growth factor-1 (Igf) 1 , and tumor necrosis factor alpha (Tnf $\alpha$ ) in WT and Dr $3^{-1-}$ intestinal are shown and represented as mean \pm s.d. $(n=3) .{ }^{\star} P<0.05,{ }^{* \star} P<0.01$. 
Dr3 in WT but not in Dr3-deficient myofibroblasts, indicating that Dr3 was expressed on $\alpha$ SMA-positive primary intestinal fibroblasts (Figure 5b).

To determine whether intestinal fibroblasts could respond to direct Tlla stimulation, changes in the expression of collagen (Col1a2, marker for fibroblast function) and Il31Ra (Il31Ra is expressed on fibroblasts) were measured with the addition of exogenous Tl1a protein. Results showed a Tl1a dose-dependent increase in the expression of Colla2 and Il31Ra in murine primary intestinal fibroblasts ex vivo (Figure 5c). The specificity of Tl1a stimulation was demonstrated by the blunted Tl1a induction of Colla 2 and Il31Ra in Dr3 ${ }^{-/-}$murine intestinal fibroblasts ex vivo (Figure 5d). In contrast, a differential induction of Colla2 or Il31Ra was not seen using known fibroblast growth factors (Tgf $\beta$ and Igf1) or proinflammatory stimuli (Tnf $\alpha$ ) (Figure 5d). These data indicated that intestinal fibroblasts expressed Dr3 and could functionally respond to direct Tl1a signaling.

\section{DISCUSSION}

The results of this study showed that blocking Tl1a function reversed established colonic fibrosis in two murine chronic colitis models. Although the decrease in inflammation resulting from blocking Tl1a is likely responsible for a component in the reduction of fibrosis, several lines of evidence indicated that TNFSF15 could be a pro-fibrogenic gene that perpetuates the process of fibrosis independent of inflammation. First, constitutive Tlla expression in mice resulted in increased colonic collagen deposition in the absence of detectable histologic inflammation in the colon. ${ }^{14,15}$ Second, primary ureteral strictures independent of visible inflammation or nephrolithiasis were found in transgenic mice that constitutively express Tlla and the same was also found in humans with elevated TL1A levels. ${ }^{10}$ Third, there is a growing body of evidence showing TNFSF15-risk variants are associated with severe gut inflammation, intestinal fibrostenosis, and more rapid progression to surgery. ${ }^{8-11}$ In line with the role of TL1A in fibrosis, in this study Dr3, the only known receptor for Tl1a, was shown to be expressed on primary murine intestinal fibroblasts (Supplementary Figure 1C, Figures 3c,d and 5a,b) and that stimulation with Tl1a can induce the expression of Col1a2 and Il31Ra on intestinal fibroblasts (Figure $\mathbf{5 c , d}$ ). Despite the persistence of histologic inflammation with Tl1a $\mathrm{Ab}$ in the chronic DSS model (Figure 2c), collagen deposition was reversed back to the original un-inflamed baseline by the inhibition of Tl1a (Figure 1c). The reduced collagen in the chronic DSS model may partly be due to the direct anti-fibrosis effect of Tlla $\mathrm{Ab}$ rather than solely a secondary effect of reduced inflammation. In addition, blocking Tlla signaling can normalize the fibrogenic program, resulting in reduced expansion of fibroblasts and myofibroblasts and reversal of histologic fibrosis. To our knowledge, this is the only in vivo model in which blocking a single IBD-associated gene product resulted not only in mitigation of inflammation, but also in the reversal of fibrosis back to the original preinflamed level.
Fibrogenesis depends on the balance between production of ECM proteins and its enzymatic degradation. Fibroblasts are involved in fibrogenesis and the synthesis of ECM components. ${ }^{17}$ Proliferation and activation of fibroblasts to myofibroblasts lead to increased ECM production. Under normal wound healing, fibroblasts and myofibroblasts either stop proliferating, acquire an inactive phenotype, or undergo apoptosis leading to a higher rate of ECM removal. ${ }^{19,20}$ The crucial step that distinguishes normal wound healing from fibrosis may be the perpetuation of fibroblasts and myofibroblasts that results in excessive accumulation of ECM and formation of fibrotic scar. ${ }^{18}$ Our data suggested that one of the fibrogenic mechanisms of Tlla is through its direct role in increasing the number of intestinal fibroblasts and myofibroblasts. Interestingly, the reduced number of fibroblasts in Dr3deficient mice was not due to differences in proliferation or apoptosis. We hypothesize that TL1A-DR3 may have a role in fibroblast formation, either through epithelial-mesenchymal transition, endothelial-mesenchymal transition, circulating fibrocytes, or possibly other mechanisms yet to be characterized.

Molecularly, the process of fibrosis results from the complex interaction between mediators involved in the fibrogenic program (Tgf $\beta$, Ctgf, Igf1, Pten) and ECM-remodeling factors (Mmp and Timp). ${ }^{17}$ The profibrotic effects of Tgf $\beta$ and Igf1 are numerous and include expansion and activation of fibroblasts, collagen synthesis, and ECM remodeling. ${ }^{18}$ The reduced Tgf $\beta$ and Igfl expression with Tl1a Ab treatment suggested decreased fibrogenesis. Ctgf, a downstream mediator of Tgf $\beta$ signaling, is involved in fibroblast and myofibroblast proliferation and ECM synthesis. Reduced numbers of fibroblasts, myofibroblasts, and collagen synthesis with Tl1a Ab administration may have been in part due to reduced Ctgf expression.

IL31 signals through the heterodimeric receptor IL31RA and oncostatin $\mathrm{M}$ receptor, and has been linked with the development of atopic dermatitis and allergic asthma. ${ }^{21-23}$ The role of IL31RA in fibrosis has not been directly demonstrated, but is implicated by its expression in fibroblasts and myofibroblasts $^{24-26}$ and from a recent report showing that IL31RA is upregulated in aldosterone-induced cardiac fibrosis. ${ }^{27}$ IL31 has been shown to stimulate the secretion of inflammatory cytokines, chemokines, and Mmps in human colonic subepithelial myofibroblasts. $^{26}$ The data in this report suggest that Il31Ra, a Tl1a-regulated protein in primary intestinal fibroblasts, might play a role in fibrosis via production of inflammatory mediators or ECM remodeling.

Although Tlla Ab treatment led to reduced Mmp, a major mediator for ECM degradation, the expression of Timp was also reduced. Decreased expression of both Mmp and Timp with Tlla Ab may result in the removal of the fibrotic ECM component while minimizing the unwanted effect of excessive Mmp activity, including tissue injury, which may ultimately lead to fibrosis. ${ }^{28-31}$

Although Dr3, the only known receptor for Tl1a, is expressed on an $\sim 25 \%$ of vimentin ${ }^{+} \alpha \mathrm{SMA}^{+}$myoflbroblasts (Supplementary Figure S1C), this represents a small proportion of the total intestinal fibroblasts in mice with colitis $(\sim 2-4 \%)$ and 
without colitis $(\sim 1-2 \%)$; thus, raising the issue of biological relevance of direct Tl1a-Dr3 signaling in intestinal fibrosis. In addition, the relative contribution of direct (effect on fibrosis independent of inflammation) and indirect (effect on fibrosis through inflammation) fibrogenic effects of Tlla was not determined in this study. Constitutive Dr3 knockout in Ragsufficient or -deficient genetic background would still not separate the relative contribution of Tl1a-Dr3 signaling on fibroblast from other inflammatory cells that also express Dr3. One approach to assess the biological relevance of direct Tl1aDr3 signaling on fibroblasts in intestinal fibrosis would be to create mice with tissue-specific Dr3 deficency in fibroblasts, but not in other cell types. The fibroblast-specific Dr3-knockout mouse would enable assessment of the in vivo fibrogenic role of Tl1a-Dr3 signaling on fibroblasts independent from its role inflammatory cells.

Existing medical therapies that target intestinal inflammation have a relatively poor record for treating $\mathrm{CD}$ complications such as fibrostenosis and altering the natural history of $\mathrm{CD} .{ }^{32-34}$ Novel therapies are needed that target both inflammation and fibrosis. Tlla Ab is successful in murine experimental models in mitigation of intestinal inflammation and reversal of fibrosis back to baseline levels. Targeting the TL1A signaling pathway may lead to a novel class of therapeutics that could not only treat inflammation, but also reverse the fibrogenic process and alter the natural history of $\mathrm{CD}$.

\section{METHODS}

Induction of chronic colitis and treatment. C57BL/6J mice were purchased from the Jackson Laboratory (Bar Harbor, ME). Chronic DSS colitis using four cycles of $2.5 \%$ DSS in drinking water was performed as described. ${ }^{10}$ In the adoptive transfer model, colitis was induced by intraperitoneal injection of $500,000 \mathrm{CD} 4^{+} \mathrm{CD} 45 \mathrm{RB}$ hi naïve T cells isolated from WT mice to Rag1 ${ }^{-1-}$ mice. Hamster anti-mouse Tl1a Ab (12F6A, TEVA, North Wales, PA) that specifically blocked the function of Tl1a but did not mediate depletion of activated T cells (data not shown) was used. For the adoptive transfer model, Tlla Ab or control immunoglobulin G (Leinco Technologies, St Louis, MO) was injected into mice intraperitoneally twice per week beginning on day 29 (Figure 1a, left panel). For the chronic DSS model, Tlla Ab or control IgG Ab was injected intraperitonealy twice per week beginning on day 15 (Figure 1a, right panel). Baseline controls (Rag Co or WT Co) were mice analyzed before DSS treatment or adoptive transfer of naive T cells. Pre-treatment (Pre-Tx) controls were mice analyzed at day 14 for the chronic DSS model and day 28 for the adoptive transfer model. Treatment groups were mice analyzed at day 28 for the chronic DSS model and at day 56 for the adoptive transfer model (Figure 1a). All mice were maintained under specific pathogen-free conditions in the Animal Facility at Cedars-Sinai Medical Center (CSMC, Los Angeles, CA). This study was carried out in strict accordance with the Guide for the Care and Use of Laboratory Animals of the National Institutes of Health. Animal studies were approved by the CSMC Animal Care and Use Committee (protocol 3813).

DAI and histopathological analyses. DAI was calculated by scoring from 0 to 4 abnormalities regarding changes in body weight ( 0 , no weight loss; $1,1-5 \%$ weight loss; $2,5-10 \%$ weight loss; $3,10-15 \%$ weight loss; 4 , more than $15 \%$ weight loss), stool consistency ( 0 , firm dry stool; 1 , moist stool; 2 , soft adherent stool; 3 , large soft pliable stool; 4, liquid stool), stool blood performed on Hemoccult Sensa (Beckman Coulter, Brea, CA; 0, no color; 1 , flecks of blue; 2 , up to $50 \%$ blue; 3 , more than $50 \%$ blue; 4 , gross red blood) and summing the results. ${ }^{10}$ DAI score was determined every other day for the DSS model and twice a week for the adoptive transfer model. Tissue samples were processed and stained with hematoxylin and eosin by the CSMC Histology-Core. Sirius red staining was performed using the NovaUltra Sirius Red Stain Kit according to the manufacturer's protocol (IHC World, Woodstock, $\mathrm{MD})$. Immunofluorescent stain was performed on $4-\mu \mathrm{m}$ frozen sections fixed with $10 \%$ formalin, blocked in $10 \%$ bovine serum albumin, $0.1 \%$ Triton X-100 TBST, and stained overnight at $4{ }^{\circ} \mathrm{C}$ with primary antibodies: rabbit polyclonal $\alpha$ SMA Ab (Abcam, Cambridge, MA) at 1:100 dilution, anti-mouse Dr3 Ab conjugated with phycoerythrin (Biolegend, San Diego, CA) at 1:50 dilution, and $\alpha$-Vimentin Ab (Covance, San Diego, CA) at 1:2,000 dilution. The next day, secondary $\mathrm{Ab}$ was added for $2 \mathrm{~h}$ at room temperature with donkey $\alpha$-rabbit IgG and goat $\alpha$-chicken IgY (Abcam) and goat $\alpha$-chicken IgY\&L (Abcam) secondary antibodies at 1:500 dilution. Histopathological scores were assigned in a blinded manner by two trained animal pathologists (DQS and JC) as described. ${ }^{10}$ Area of collagen deposition was determined using the Image 1.47 software package. ${ }^{35}$ The numbers of fibroblasts and myofibroblasts were quantitated from colonic mucosa and submucosa using a Leica TCS SP spectral confocal microscope (Buffalo Grove, IL).

Sircol assay. Acid and pepsin-soluble collagen from the mid-colon (3-mm piece) were measured using the Sircol Soluble Collagen Assay according to the manufacturer's protocol (Biocolor, Northern Ireland, UK). Acid and pepsin-soluble collagen were measured using a colorimetric absorbance using the 550-nm filter and concentration determined using the collagen standard provided in the Sircol Soluble Collagen Assay Kit. Recovered mid-colon collagen is normalized to total acid and pepsin-soluble protein measured using Bradford Protein Assay Kit according to the manufacturer's protocol (Thermo Scientific, Rockford, IL).

Generation of $\mathrm{Dr}^{-/-}$mice. Cloning of Dr3-targeting vector and generation of $\mathrm{Dr} 3^{+/-}$founder mice were performed in collaboration with genOway (genOway, Lyon, France). Briefly, Dr3 endogenous locus containing $1.5 \mathrm{~kb}$ upstream of exon 1 and $3 \mathrm{~kb}$ downstream of exon 8 was generated by PCR amplification using genomic DNA from $\mathrm{C} 57 \mathrm{BL} / 6 \mathrm{~J}$ mice and cloned into the pCR4-TOPO vector (Invitrogen, Carlsbad, CA). Subsequently, two loxP sites were inserted flanking Dr3 exons 2-5 (Supplementary Figure S1A). Positive selection neomycin gene flanked by flippase recognition target sites was inserted to the intron between exon 1 and 2 to generate the targeting vector (Supplementary Figure S1A). Every step of the cloning process was validated through restriction enzyme analysis and sequencing. The Dr3 gene-targeting construct was linearized and electroporated into genOway proprietary embryonic stem cells with C57BL/6J background. Homologous recombinants were selected by G418 and confirmed by Southern blot analysis. Embryonic stem clones with correct $5^{\prime}$ and $3^{\prime}$ recombination were microinjected into C57BL/6J blastocysts and introduced into pseudopregnant C57BL/6J mice. Male chimeric offspring were bred to obtain germ line mutant mice, which were then bred to Flpe-delete mouse strain to remove the neomycin cassette, then bred to Cre-delete mice to excise the loxP-flanked sequences (Supplementary Figure S1A), confirmed by Southern blot, and maintained on the C57BL/6J genetic background.

Expression analysis. Total RNA was isolated using RNeasy Microarray Tissue Mini Kit (Qiagen, Valencia, CA), and reversetranscription PCR (RT-PCR) was performed using RT2 HT First Strand and gene expression was measured using the RT2 Custom Fibrosis Array CAPM11248 (Qiagen, Valencia, CA) kits as per the manufacturer's protocols. Validated Dr3 qPCR assay Mm.PT.51. 17321439, Il31Ra qPCR assay Mm.PT.56a.32787326, Tl1a qPCR assay Mm.PT.51.17321439, Col1a2 qPCR assay Mm.PT.51.5206680, and $\beta$-actin qPCR assay Mm.PT.39a.22214843 were purchased from IDT Technologies (Skokie, IL). 
Cell isolation, culture, flow cytometry, and cell sorting. Isolation and culture of lamina propria mononuclear cells and mesenteric lymph node cells were carried out as previously reported. ${ }^{14}$ The whole colon and the distal $10 \mathrm{~cm}$ of the ileum were used for lamina propria mononuclear cells isolation. $\mathrm{CD}^{+}{ }^{+} \mathrm{T}$ cells were isolated from spleen and mesenteric lymph node by negative selection (StemCell Technologies, Vancouver, $\mathrm{BC}$, Canada). mesenteric lymph node, lamina propria mononuclear cells, and $\mathrm{CD}^{+}{ }^{+} \mathrm{T}$ cells were cultured in 96-well round-bottom plates at $1.5 \times 10^{6}$ cells $\mathrm{ml}^{-1}$ of $\mathrm{T}$ cell medium (RPMI 1640 containing $2 \mathrm{mmoll}^{-1}$ glutamine, $25 \mathrm{mmoll}^{-1}$ HEPES, $10 \%$ fetal bovine serum, $100 \mu \mathrm{M}$ nonessential amino acid, $1 \mathrm{~mm}$ sodium pyruvate, $50 \mu \mathrm{M}$ $\beta$-mercaptoethanol, $50 \mu \mathrm{g} \mathrm{ml}^{-1}$ gentamicin) under conditions indicated in Supplementary Figure S1 with Il12 $\left(5 \mathrm{ng} \mathrm{ml}^{-1}\right)$, Tnf $\alpha\left(5 \mathrm{ng} \mathrm{ml}^{-1}\right)$, Il6 (100 $\left.\mathrm{ng} \mathrm{ml}^{-1}\right)$, anti-CD3e $\left(0.5 \mu \mathrm{g} \mathrm{ml}^{-1}, 145-2 \mathrm{C} 11\right)$, anti-CD28 $\left(1 \mu \mathrm{g} \mathrm{ml}^{-1}, 37.51\right)$, Tlla Ab $\left(50 \mu \mathrm{g} \mathrm{ml}^{-1}, 12 \mathrm{~F} 6 \mathrm{~A}\right.$, TEVA, North Wales, PA), and control isotype immunoglobulin $\mathrm{G}\left(50 \mu \mathrm{g} \mathrm{ml}^{-1}\right.$, Leinco Technologies). Cells and media were harvested $48 \mathrm{~h}$ after culture and assessed for Ifn $\gamma$ production via enzyme-linked immunosorbent assay kit (eBioscience, San Diego, CA), proliferation and apoptosis assay as described below.

Mouse primary colonic fibroblasts were isolated from colon that were incubated in $1 \mathrm{~mm}$ dithiothreitol (Fisher Scientific, Tustin, CA), $37^{\circ} \mathrm{C}, 15 \mathrm{~min}$, and then $1 \mathrm{~mm}$ dithiothreitol with $5 \mathrm{~mm}$ EDTA (Promega, Madison, WI), $37^{\circ} \mathrm{C}, 30 \mathrm{~min}$. The remaining colonic tissues were rinsed by $1 \times$ Hank's balanced salt solution (Corning Cellgro, Swedesboro, NJ), minced, and then digested for $30 \mathrm{~min}$ at $37^{\circ} \mathrm{C}$ with $1.5 \mathrm{mg} \mathrm{ml}^{-1}$ Collagenase II (Worthington, Lakewood, NJ), $0.3 \mathrm{mg} \mathrm{ml}^{-1}$ DNase I, and $3 \mathrm{mg} \mathrm{ml}^{-1}$ Hyaluronidase (Sigma, St Louis, MO) in DMEM (Corning Cellgro, Swedesboro, NJ). The isolated cells were cultured in DMEM supplemented with $10 \%$ fetal calf serum, penicillin/streptomycin $\left(100 \mathrm{IU} \mathrm{ml}^{-1}\right)$, Fungizone $\left(0.5 \mu \mathrm{g} \mathrm{ml}^{-1}\right)$. The number of fibroblasts was counted using a hemocytometer and represented as total fibroblast recovered per colon. The criteria to score cells as fibroblast are based upon morphology and positive vimentin staining. Primary intestinal fibroblasts were used at passage 2. Intracellular staining and antibodies used were described previously. ${ }^{14,36}$ Growth factors Tgf $\beta 100 \mathrm{ng} \mathrm{ml}^{-1}$ and Igf1 $10 \mathrm{ng} \mathrm{ml}^{-1}$ (Cell Signaling, Danvers, MA) and cytokines Tl1a 10-200 or $100 \mathrm{ng} \mathrm{ml}^{-1}$ (R\&D Systems, Minneapolis, MN), and Tnf $\alpha 5 \mathrm{ng} \mathrm{ml}^{-1}$ (Biolegend, San Diego, CA) were added to passage 2 fibroblasts for $4 \mathrm{~h}$ before harvesting for expression analysis.

For flow cytometry, cells were acquired on a LSR II flow-cytometer (BD Biosciences, San Jose, CA) and analyzed using FlowJo analysis software. For cell sorting, cells were sorted using the MoFlo Cell Sorter with the assistance from Flow Cytometry Core Facility at CSMC.

Ex vivo intestinal fibroblast proliferation and apoptosis assay. Primary intestinal fibroblasts were isolated and stained with CellTrace Violet (Invitrogen, Carlsbad, CA) as per the manufacturer's instructions. Stained cells were then incubated with $100 \mathrm{ng} \mathrm{ml}^{-1}$ of Tlla in DMEM supplemented with $10 \%$ fetal calf serum, penicillin/streptomycin $\left(100 \mathrm{IU} \mathrm{ml}^{-1}\right)$, and Fungizone $\left(0.5 \mu \mathrm{g} \mathrm{ml}^{-1}\right)$. After $48 \mathrm{~h}$, cultured intestinal fibroblasts were stained using Annexin V Apoptosis Detection Kit (eBioscience, San Diego, CA) as per the manufacturer's instructions. After Annexin V stain, fibroblasts were harvested, washed, and fixed with $2 \%$ paraformaldehyde and subjected to flow cytometric analysis with BD LSR II flow-cytometer (BD Biosciences) and analyzed by FlowJo software (Ashland, OR).

Statistical analysis. Data are presented as the mean \pm s.d. Comparison between two groups was performed by a two-tailed Fisher's Exact test for categorical variables and Student's $t$-test for continuous variables. Parametric and non-parametric tests were used depending on the fulfillment of the test assumptions. Comparison between three groups was done using analysis of variance, followed by pair wise post hoc analysis with Turkey's HSD and Behrens-fisher-Test correction for the multiple comparisons. $P<0.05$ was considered significant.
SUPPLEMENTARY MATERIAL is linked to the online version of the paper at http://www.nature.com/mi

\section{ACKNOWLEDGMENTS}

We thank Jyh-Yau Chang for assistance in flow cytometry and Loren Karp for critical reading of the manuscript. This work was supported by USPHS grant DK056328 (S.R.T.), NIH K08 Career Development Award DK093578 (D.Q.S.), investigator initiated Grant from TEVA Pharmaceuticals (S.R.T.), and the F. Widjaja Foundation Inflammatory Bowel \& Immunobiology Research Institute. The project described was also supported by the National Center for Advancing Translational Sciences, Grant UL1TR000124 (D.Q.S.).

\section{DISCLOSURE}

The author declared no conflict of interest.

c 2014 Society for Mucosal Immunology

\section{REFERENCES}

1. Cosnes, J. et al. Long-term evolution of disease behavior of Crohn's disease. Inflamm. Bowel Dis. 8, 244-250 (2002).

2. Peyrin-Biroulet, L. \& Lemann, M. Review article: remission rates achievable by current therapies for inflammatory bowel disease. Aliment. Pharmacol. Ther. 33, 870-879 (2011).

3. Johnson, L.A., Luke, A., Sauder, K., Moons, D.S., Horowitz, J.C. \& Higgins, P.D. Intestinal fibrosis is reduced by early elimination of inflammation in a mouse model of IBD: impact of a "Top-Down" approach to intestinal fibrosis in mice. Inflamm. Bowel Dis. 18, 460-471 (2012).

4. Hardie, W.D., Le Cras, T.D., Jiang, K., Tichelaar, J.W., Azhar, M. \& Korfhagen, T.R. Conditional expression of transforming growth factoralpha in adult mouse lung causes pulmonary fibrosis. Am. J. Physiol. Lung Cell. Mol. Physiol. 286, L741-L749 (2004).

5. Lavaud, S. et al. Inflammation is probably not a prerequisite for renal interstitial fibrosis in normoglycemic obese rats. Am. J. Physiol. Renal Physiol. 280, F683-F694 (2001).

6. Koon, H.W. et al. Substance P modulates colitis-associated fibrosis. Am. J. Pathol. 177, 2300-2309 (2010).

7. Shih, D.Q. et al. Insights into TL1A and IBD pathogenesis. Adv. Exp. Med. Biol. 691, 279-288 (2011).

8. Picornell, Y., Mei, L., Taylor, K., Yang, H., Targan, S.R. \& Rotter, J.I. TNFSF15 is an ethnic-specific IBD gene. Inflamm. Bowel Dis. 13, 1333-1338 (2007).

9. Michelsen, K.S. et al. IBD-associated TL1A gene (TNFSF15) haplotypes determine increased expression of TL1A protein. PLoS One 4, e4719 (2009).

10. Barrett, R. et al. Constitutive TL1A expression under colitogenic conditions modulates the severity and location of gut mucosal inflammation and induces fibrostenosis. Am. J. Pathol. 180, 636-649 (2012).

11. Hirano, A. et al. Association study of 71 European Crohn's disease susceptibility loci in a Japanese population. inflamm. Bowel Dis. 19, 526-533 (2013).

12. Meylan, F. et al. The TNF-family cytokine TL1A drives IL-13dependent small intestinal inflammation. Mucosal Immunol. 4, 172-185 (2011).

13. Taraban, V.Y. et al. Sustained TL1A expression modulates effector and regulatory $\mathrm{T}$-cell responses and drives intestinal goblet cell hyperplasia. Mucosal Immunol. 4, 186-196 (2011).

14. Shih, D.Q. et al. Constitutive TL1A (TNFSF15) expression on lymphoid or myeloid cells leads to mild intestinal inflammation and fibrosis. PLoS One 6, e16090 (2011)

15. Zheng, L. et al. Sustained Tl1a expression on both lymphoid and myeloid cells leads to mild spontaneous intestinal inflammation and fibrosis. Eur. J. Microbiol. Immunol. 3, 11-20 (2013).

16. Takedatsu, H. et al. TL1A (TNFSF15) regulates the development of chronic colitis by modulating both T-helper 1 and T-helper 17 activation. Gastroenterology 135, 552-567 (2008).

17. Wynn, T.A. \& Ramalingam, T.R. Mechanisms of fibrosis: therapeutic translation for fibrotic disease. Nat. Med. 18, 1028-1040 (2012). 
18. Speca, S., Giusti, I., Rieder, F. \& Latella, G. Cellular and molecular mechanisms of intestinal fibrosis. World J. Gastroenterol. 18, 3635-3661 (2012).

19. Iredale, J.P. et al. Mechanisms of spontaneous resolution of rat liver fibrosis. Hepatic stellate cell apoptosis and reduced hepatic expression of metalloproteinase inhibitors. J. Clin. Investig. 102, 538-549 (1998).

20. Kisseleva, T. et al. Myofibroblasts revert to an inactive phenotype during regression of liver fibrosis. Proc. Natl Acad. Sci. USA 109, 9448-9453 (2012).

21. Dillon, S.R. et al. Interleukin 31, a cytokine produced by activated T cells, induces dermatitis in mice. Nat. Immunol. 5, 752-760 (2004).

22. Bilsborough, J., Mudri, S., Chadwick, E., Harder, B. \& Dillon, S.R. IL-31 receptor (IL-31RA) knockout mice exhibit elevated responsiveness to oncostatin M. J. Immunol. 185, 6023-6030 (2010).

23. Lei, Z. et al. SCF and IL-31 rather than IL-17 and BAFF are potential indicators in patients with allergic asthma. Allergy 63, 327-332 (2008).

24. Wong, C.K., Leung, K.M., Qu, H.N., Chow, J.Y., Choi, A.O. \& Lam, C.W. Activation of eosinophils interacting with dermal fibroblasts by pruritogenic cytokine IL-31 and alarmin IL-33: implications in atopic dermatitis. PLoS One 7, e29815 (2012).

25. Jawa, R.S. et al. Regulated expression of the IL-31 receptor in bronchial and alveolar epithelial cells, pulmonary fibroblasts, and pulmonary macrophages. J. Interferon Cytokine Res. 28, 207-219 (2008).

26. Yagi, Y. et al. Interleukin-31 stimulates production of inflammatory mediators from human colonic subepithelial myofibroblasts. Int. J. Mol. Med. 19, 941-946 (2007).

27. Chen, J.S. et al. Screening for genes associated with cardiac fibrosis induced by aldosterone. Xi Bao Yu Fen Zi Mian Yi Xue Za Zhi 28, 350-353 (2012).
28. Louis, E. et al. Increased production of matrix metalloproteinase-3 and tissue inhibitor of metalloproteinase- 1 by inflamed mucosa in inflammatory bowel disease. Clin. Exp. Immunol. 120, 241-246 (2000).

29. Gao, Q. et al. Expression of matrix metalloproteinases-2 and -9 in intestinal tissue of patients with inflammatory bowel diseases. Dig. Liver Dis. 37, 584-592 (2005).

30. Heuschkel, R.B., MacDonald, T.T., Monteleone, G., Bajaj-Elliott, M., Smith, J.A. \& Pender, S.L. Imbalance of stromelysin-1 and TIMP-1 in the mucosal lesions of children with inflammatory bowel disease. Gut 47, 57-62 (2000).

31. Stawowy, P. et al. Regulation of matrix metalloproteinase MT1-MMP/ MMP-2 in cardiac fibroblasts by TGF-beta1 involves furin-convertase. Cardiovasc. Res. 63, 87-97 (2004).

32. Andres, P.G. \& Friedman, L.S. Epidemiology and the natural course of inflammatory bowel disease. Gastroenterol. Clin. North Am. 28, 255-281. vii (1999).

33. Vermeire, S., van Assche, G. \& Rutgeerts, P. Review article: altering the natural history of Crohn's disease-evidence for and against current therapies. Aliment. Pharmacol. Ther. 25, 3-12 (2007).

34. Cosnes, J., Gower-Rousseau, C., Seksik, P. \& Cortot, A. Epidemiology and natural history of inflammatory bowel diseases. Gastroenterology 140 , 1785-1794 (2011).

35. Vallance, B.A. et al. TGF-beta1 gene transfer to the mouse colon leads to intestinal fibrosis. Am. J. Physiol. Gastrointest. Liver Physiol. 289, G116-G128 (2005).

36. Fang, L., Adkins, B., Deyev, V. \& Podack, E.R. Essential role of TNF receptor superfamily 25 (TNFRSF25) in the development of allergic lung inflammation. J. Exp. Med. 205, 1037-1048 (2008). 